\title{
Mindfulness in Relation to Diet Quality in Adults with Type 1 and Type 2 Diabetes: Results from Diabetes MILES-The Netherlands
}

\author{
Shengxin Liu' ${ }^{1}$ • Ivan Nyklíček ${ }^{2}$. Frans Pouwer ${ }^{3,4} \cdot$ Jane Speight $^{5,6} \cdot$ Mariska Bot $^{7} \cdot$ Giesje Nefs $^{8,9}$. \\ Sabita S. Soedamah-Muthu' ${ }^{2,10}$
}

Accepted: 7 September 2021 / Published online: 18 September 2021

(c) The Author(s) 2021

\begin{abstract}
Objectives The objectives of this study were to investigate the associations of dispositional mindfulness and its facets with diet quality in Dutch adults with type 1 diabetes and type 2 diabetes and to evaluate the potential mediating role of emotional distress in these associations.

Methods This was a cross-sectional study using data from the Diabetes MILES-The Netherlands. In total, 296 adults with type 1 diabetes and 364 with type 2 diabetes completed questionnaires. We assessed dispositional mindfulness by the Five Facet Mindfulness Questionnaire-Short Form, including total mindfulness and five facets: observing, describing, acting with awareness, being non-judgmental, and being non-reactive, and diet quality by the Dutch Healthy Diet 2015-index. Linear regression models were used to estimate the association. Mediation effects of depressive symptoms, anxiety symptoms, and diabetes-related distress were evaluated using the PROCESS macro with bias-corrected bootstrapped confidence intervals. Results Higher scores on the total mindfulness $(\beta=0.14, p=0.02)$ and the observing facet $(\beta=0.15, p=0.01)$ were significantly associated with higher diet quality in people with type 1 diabetes only, after adjustment for demographic factors. These associations were not mediated by depressive symptoms, anxiety symptoms, or diabetes-related distress. In adults with type 1 diabetes (but not type 2), higher dispositional mindfulness levels were associated with more optimal diet quality. Conclusions Higher mindfulness in general and observing in particular were related to a healthier diet with higher quality in Dutch adults with type 1 diabetes. Further longitudinal studies are warranted to understand the temporal relationship between mindfulness and diet quality.
\end{abstract}

Keywords Type 1 diabetes $\cdot$ Type 2 diabetes $\cdot$ Mindfulness $\cdot$ Diet quality $\cdot$ Following of dietary guidelines

Diabetes mellitus ranks among the most common chronic conditions and the prevalence is still fast increasing worldwide. The global estimate for the number of adults with diabetes was 463 million in 2019, and this number is expected

Shengxin Liu

Shengxin.liu@ki.se

1 Department of Medical Epidemiology and Biostatistics, Karolinska Institutet, Solna, Sweden

2 Center of Research on Psychological Disorders and Somatic Diseases (CoRPS), Department of Medical and Clinical Psychology, Tilburg University, Tilburg, the Netherlands

3 Department of Psychology, University of Southern Denmark, Odense, Denmark

4 STENO Diabetes Center Odense, Odense, Denmark

5 School of Psychology, Deakin University, Geelong, VIC, Australia to have grown to 700 million by 2045 (IDF Diabetes Atlas, 2019). The management of diabetes mostly consists of self-management by the person with diabetes. One of the cornerstones in diabetes self-management is diet, such as

6 The Australian Centre for Behavioural Research in Diabetes, Melbourne, VIC, Australia

7 Department of Psychiatry, Amsterdam Public Health Research Institute, Amsterdam UMC, Amsterdam, the Netherlands

8 Department of Medical Psychology, Radboud University Medical Center, Radboud Institute for Health Sciences, Nijmegen, the Netherlands

9 Diabeter, National Treatment and Research Center for Children, Adolescents and Adults With Type 1 Diabetes, Rotterdam, the Netherlands

10 Institute for Food, Nutrition and Health (IFNH), University of Reading, Reading, UK 
diets low in carbohydrates (especially in type 2 diabetes) and diets high in vegetables and fruits (in both types of diabetes). In general, all people should aim for high-quality diets following evidence-based dietary guidelines. For example, prospective studies have shown that such healthy diets are related to $\mathrm{a} \sim 30 \%$ reduction in cardiovascular risk in people with diabetes and subsequently a lower mortality rate (Estruch et al., 2013; Ley et al., 2014; Liday \& Kirkpatrick, 2019).

For various reasons, maintaining a healthy diet is challenging for many people, also for both people with type 1 diabetes (T1DM) and type 2 diabetes (T2DM) (Ahola et al., 2012; Forouhi et al., 2018). However, the inability to follow recommendations related to a healthy diet can consequently hamper the management of blood glucose. Studies have found that several psychological aspects contribute to self-management of diabetes, including people's acceptance, mental ability to cope with the condition, and willingness to change lifestyles, including dietary habits (Glasgow et al., 1986; Richardson et al., 2001; Senécal et al., 2000; Shultz et al., 2001). Therefore, identifying psychological factors that can facilitate self-management, especially concerning following dietary recommendations, is of utmost importance in people with diabetes.

Mindfulness, which is defined as bringing one's attention to present-moment experience with acceptance (KabatZinn, 2009), has been associated with engagement in various healthy eating behaviors. This concept consists of five intercorrelated facets (Baer et al., 2006): observing (noticing or attending to internal and external stimuli, such as smells, sights, or sounds), describing (expressing internal experience with words), acting with awareness (doing things with a focus on the present moment), being non-judgmental (taking a no evaluative stance to internal feelings and thoughts), and being non-reactive (allowing feelings and thoughts to happen without reacting to them).

Evidence from systematic reviews and meta-analyses of intervention studies suggests that dispositional mindfulness, as a trait, is associated with healthier eating behaviors, such as less binge and emotional eating (Barney et al., 2019; Sala et al., 2020). A recent meta-analysis demonstrated a weighted average correlation coefficient $(r)$ of $0.14(p<0.001)$ between mindfulness and healthy eating behaviors based on 24 studies with healthy individuals (Sala et al., 2020). In the same study, each facet of mindfulness was found to be positively related to more optimal healthy eating behaviors $(r=0.10-0.19$, all $p<0.01$ ). However, this meta-analysis did not involve studies focusing on people with diabetes. Our research group has previously reported similar findings regarding the mindfulness and eating behaviors relationship in people with either T1DM or T2DM, where a higher level of mindfulness was observed to be associated with less emotional eating $(\beta=-0.20$, $p<0.001$ ) (Tak et al., 2015). Moreover, it has been suggested that mindfulness-based interventions could reduce disordered eating and lessen hunger susceptibility in people with T2DM (Mason et al., 2016; Miller, 2017; Miller et al., 2014). Nevertheless, changes in eating behaviors do not necessarily reflect the comprehensive diet quality, which also depends on the types of consumed food (e.g., refined grain versus whole grain), amount, and frequency of intake.

A higher mindfulness score has been associated with less intake of sugar and energy-dense foods (Beshara et al., 2013; Jordan et al., 2014) and increased intake of fruits and vegetables (Miller et al., 2014) in people with T2DM. Still, it remains unclear whether mindfulness could benefit both people with T1DM and T2DM in improving their diet quality since a single food item, as studied previously, cannot provide a full picture of the diet pattern (Patterson et al., 1994). Studies in normal-weight and over-weight populations have observed tendencies towards choosing healthier foods as a result of improved mindfulness. A recently published study showed that mindfulness was related to more optimal diet quality, consistent with the American Dietary Guideline and evaluated using the diet index in middle-aged adults (Donofry et al., 2020). The usage of the diet index provided a more comprehensive overview of the diet quality. However, mindfulness was examined as a unidimensional construct without investigating each mindfulness facet in detail.

With respect to potential underlying pathways between mindfulness and diet quality, the role of emotional distress should not be disregarded. We have previously found that mindfulness is negatively related to depressive symptoms, anxiety symptoms, and diabetes-specific distress in people with diabetes (van Son et al., 2015). These emotional distress factors have also been associated with increased intake of foods with high fat and sugar and, subsequently, increased calorie intake with less optimal diet quality (Barrington et al., 2014; Mikolajczyk et al., 2009).

The aim of this study was to investigate the associations between dispositional mindfulness and diet quality in Dutch adults with T1DM or T2DM. We hypothesized that a higher level of mindfulness is related to greater diet quality. In the light of previous research in other populations (Sala et al., 2020), we hypothesized that each mindfulness facet is positively associated with diet quality. In addition, we evaluated the potential mediating role of emotional distress in these associations.

\section{Methods}

\section{Participants}

The present study was a part of the Diabetes MILES (Management and Impact for Long-term Empowerment and 
Success)-The Netherlands, which was a national, online cross-sectional survey among adults with T1DM or T2DM. The survey was conducted in the Netherlands during fall 2011, and detailed descriptions have been published elsewhere (Nefs et al., 2012).

A total of 660 participants completed the core questionnaires and the randomly assigned mindfulness module, including 296 participants with T1DM and 364 participants with T2DM. As shown in Table 1, participants with T2DM were older and more likely to have a lower educational level and to be unemployed, compared to participants with T1DM. They also tended to have higher BMI and lower $\mathrm{HbA}_{1 \mathrm{c}}$ (both medium effect sizes). Diabetes duration was also visibly and statistically shorter in participants with T2DM.

\section{Procedures}

Participants were recruited via several diabetes-related media channels. A total of 3,228 participants completed the core set of questionnaires, including items about general health, lifestyle behavior, and emotional wellbeing. They were then randomly allocated to complete one out of five complementary questionnaire subsets. The present study used data of 660 participants who were assigned to and completed the subset of questionnaires on mindfulness. The Diabetes MILES-The Netherlands was approved by the Psychological Research Ethics Committee of Tilburg University (EC-2011 5), and digital informed consent was obtained from all participants.

\section{Measures}

\section{Demographic and Clinical Variables}

Information on demographic and clinical variables was obtained through self-reported data from the core survey. For the present analysis, demographic variables included gender (male/female), age (years), and paid employment (yes/no), marital status (single/partner), and ethnicity (Dutch/ethnic minority). Educational level was categorized as low (no education, elementary, lower secondary, and low vocational education), intermediate (intermediate vocational or higher secondary education), and high (high vocational or university education). Body mass index (BMI) was calculated based on self-reported weight and height. Information on diabetes type, duration of diabetes, and the most recent measurement of glycated hemoglobin $\left(\mathrm{HbA}_{1 \mathrm{c}}\right)$ were also obtained via this questionnaire.
Table 1 Descriptive characteristics of the study participants $(n=660)$, stratified by diabetes type

\begin{tabular}{|c|c|c|c|}
\hline & Type 1 diabetes & Type 2 diabetes & Effect size* \\
\hline Total $N^{\mathrm{a}}$ & 296 & 364 & \\
\hline Males, \% (n) & $42.2(125)$ & $51.4(187)$ & 0.09 \\
\hline Age, years, mean $(\mathrm{SD})^{\mathrm{b}}$ & $47.3(15.0)$ & $61.3(9.4)$ & $1.14^{* *}$ \\
\hline With partner, $\%(n)^{\mathrm{c}}$ & $17.9(53)$ & $19.0(69)$ & 0.01 \\
\hline Ethnic minority, \% (n) & $1.4(4)$ & $2.8(10)$ & 0.05 \\
\hline Paid employment, \% ( $n)$ & $64.9(192)$ & $38.2(139)$ & $0.27 * *$ \\
\hline Education level, \% (n) & & & $0.15^{* *}$ \\
\hline High & $51.4(152)$ & $36.8(134)$ & \\
\hline Middle & $30.4(90)$ & $35.2(128)$ & \\
\hline Low & $18.2(54)$ & $28.0(102)$ & \\
\hline Body mass index, $\mathrm{kg} / \mathrm{m}^{2}$, mean $(\mathrm{SD})^{\mathrm{d}}$ & $25.5(5.7)$ & $29.7(6.0)$ & $0.73 * *$ \\
\hline Diabetes duration, years, mean (SD) & $23.6(14.9)$ & $10.8(7.6)$ & $1.12 * *$ \\
\hline $\mathrm{HbA}_{1 \mathrm{c}} \mathrm{mmol} / \mathrm{mol}$, mean $(\mathrm{SD})^{\mathrm{e}}$ & $58.7(11.9)$ & $53.3(11.5)$ & $0.46^{* *}$ \\
\hline
\end{tabular}

*Cohen's $d$ for continuous variables and Cramer's $V$ for categorical variables

${ }^{* *}$ Statistically significant, $p$-value $<0.05$

${ }^{a}$ Number of participants fulfilled inclusion criteria

Information on missing data:

${ }^{\mathrm{b}}$ Information on paid employment was missing for one participant with type 1 diabetes

${ }^{\mathrm{c}}$ Information on age was missing for 10 participants ( 5 type 1 diabetes and 5 type 2 diabetes)

${ }^{\mathrm{d}}$ Information on body mass index was missing for 12 participants ( 6 type 1 diabetes and 6 type 2 diabetes)

${ }^{\mathrm{e}}$ Information on the latest $\mathrm{HbA}_{1 \mathrm{c}}$ value was missing for 179 participants (51 type 1 diabetes and 128 type 2 diabetes)

$N$, number of study participants; $S D$, standard deviation; \%, percentage; $\mathrm{kg}$, kilogram; $\mathrm{m}^{2}$, meters squared; $\mathrm{HbAlc}$, glycated hemoglobin; $\mathrm{mmol} / \mathrm{mol}$, millimoles per mole 


\section{Dispositional Mindfulness}

Dispositional mindfulness was evaluated by the Five Facet Mindfulness Questionnaire-Short Form (FFMQ-SF), which is a reliable 24-item version of the original 39-item FFMQ and has been validated in a community setting (Bohlmeijer et al., 2011). The five facets of mindfulness included the following: observing ( 4 items), describing (5 items), acting with awareness (5 items), being non-judgmental (5 items), and being non-reactive (5 items). Participants were asked to rate to what degree each statement is true for them, using a 5-point Likert-type scale, ranging from 1 (never or very rarely true) to 5 (very often or always true). The FFMQSF total score was calculated by summing up all 24 item scores and ranged from 24 to 120 . Higher scores indicated a higher level of dispositional mindfulness. Facet-specific scores were the sums of items for each facet, ranging from 4 to 20 for observing ( 4 items) and 5 to 25 for other facets ( 5 items). Cronbach's alpha coefficients of the FFMQ-SF total and facet-specific scores ranged from 0.75 to 0.87 (Bohlmeijer et al., 2011). In our current study sample, Cronbach's alpha was 0.75 for observing; 0.79 for describing; 0.82 for acting with awareness; 0.73 for being non-judgmental; and 0.71 for being non-reactive. Differences in alpha between T1DM and T2DM were minor, with a range of 0.72 to 0.83 in T1DM and 0.70 to 0.83 in T2DM.

\section{Dietary Assessment and Dutch Healthy Diet 2015-Index}

A 38-item Food Frequency Questionnaire (FFQ) was used to assess dietary intakes of various food items (Nefs et al., 2012). Participants were asked about the consumption of 32 food items during the month preceding participation, with a frequency of consumption categorized as " 0 days per week," "1-3 days per week," "4-5 days per week," and "6-7 days per week" (Vogtschmidt et al., 2020). For alcohol consumption, frequency categories were "none," "yes, $<1$ glass per week," "yes, 1-7 glasses per week," "yes, 8-14 glasses per week," "yes, 15-21 glasses per week," "yes, 22-28 glasses per week," "yes, 29-35 glasses per week," and "yes, 36 glasses or more per week." The portion size of each food item was not asked in the FFQ; this information was provided by two datasets kept by the National Institute for Public Health and the Environment (RIVM), namely the Dutch National Food Composition Database (NEVO) (Westenbrink et al., 2012) and the Dutch National Food Consumption Survey of 2012-2014. Total consumption quantity was calculated by multiplying the estimated portion size by frequency of consumption and presented in grams per day $(\mathrm{g} /$ day). Total energy intake (kcal/day), alcohol intake (g/day), and salt intake (mg/day) were further calculated from all dietary data based on NEVO.
The Dutch Healthy Diet 2015-index score (DHD15index score) is a validated measure of diet quality following the food-based Dutch dietary guidelines published by the Health Council of the Netherlands in 2015 (Looman et al., 2017). The detailed calculation of the DHD15-index in the current study population has been described previously (Vogtschmidt et al., 2020). In short, a total of 12 components of food groups (e.g., vegetables, fruits) were scored corresponding to the recommendations from the dietary guidelines (e.g., $>200 \mathrm{~g} /$ day). Each component has a score ranging from 0 (not following the dietary guidelines) to 10 (completely following the dietary guidelines), resulting in a total score between 0 and 120 points, with a higher value indicating a higher diet quality. A detailed description of each component and the dietary recommendations is listed in Supplemental Table 1.

\section{Emotional Distress}

Depressive symptoms were assessed using the validated 9-item Patient Health Questionnaire (PHQ-9) (Kroenke et al., 2001) and anxiety symptoms with the 7-item Generalized Anxiety Disorder Assessment (GAD-7) (Spitzer et al., 2006). Both questionnaires were developed based on diagnostic symptoms of each disorder with corresponding items rated on a 4-point Likert-type scale, ranging from 0 (not at all) to 3 (nearly every day). Item scores are summed into a total score (0-27 for PHQ-9 and 0-21 for GAD-7), with a higher score indicating a higher likelihood of depressive or anxiety disorder. A cutoff score of $\geq 10$ was used to categorize participants into those with and without moderate depressive and anxiety symptoms (Kroenke et al., 2001; Spitzer et al., 2006). Diabetes-specific distress was assessed by using the 20-item Problem Areas in Diabetes scale (PAID-20), a questionnaire that showed good validity in both T1DM and T2DM (Snoek et al., 2000). Participants were asked to rate their experiences on a 5-point Likerttype scale from 0 "not a problem" to 4 "serious problem." The total score of PAID-20 is the sum of all item scores and standardized into a 0 to 100 scale, with a higher score indicating more diabetic-specific distress.

\section{Data Analyses}

All analyses were performed separately by diabetes type because of the distinct etiology of T1DM and T2DM. Demographic and clinical characteristics of participants were calculated and presented as means (standard deviation, SD) for continuous variables or $n$ (percentage, \%) for categorical variables. Differences in characteristics between T1DM and T2DM were examined using Student's $t$-test for continuous variables and chi-square test for categorical variables. The effect size of between-group differences was measured 
Table 2 Description of FFMQ total and facet-specific scores, the Dutch Healthy Diet 2015-index score and emotional distress assessment scores, stratified by diabetes type

\begin{tabular}{|c|c|c|c|c|c|}
\hline & $\begin{array}{l}\text { Potential score } \\
\text { ranges }\end{array}$ & Type 1 diabetes & Type 2 diabetes & Cohen's $d^{*}$ & $p$-value \\
\hline Total $N^{\mathrm{a}}$ & & 296 & 364 & & \\
\hline \multicolumn{6}{|l|}{ Mindfulness (FFMQ-SF), mean (SD) } \\
\hline Total & $24-120$ & $83.9(12.1)$ & $84.3(11.6)$ & 0.04 & 0.63 \\
\hline Observing & $4-20$ & $13.7(3.6)$ & $13.5(3.7)$ & 0.06 & 0.42 \\
\hline Describing & $5-25$ & $18.3(4.1)$ & $18.3(3.8)$ & 0.003 & 0.97 \\
\hline Acting with awareness & $5-25$ & $19.0(3.9)$ & $19.5(3.7)$ & 0.15 & 0.06 \\
\hline Being non-judgmental & $5-25$ & $17.6(3.9)$ & $17.8(4.0)$ & 0.04 & 0.64 \\
\hline Being non-reactive & $5-25$ & $15.3(3.8)$ & $15.3(3.9)$ & 0.004 & 0.96 \\
\hline DHD15-index score, mean (SD) & $0-120$ & $83.7(13.7)$ & $84.5(12.7)$ & 0.06 & 0.46 \\
\hline \multicolumn{6}{|l|}{ Emotional distress } \\
\hline Depressive symptoms (PHQ-9), mean (SD) & $0-27$ & $4.5(4.8)$ & $4.4(4.9)$ & 0.03 & 0.74 \\
\hline Anxiety symptoms (GAD-7), mean (SD) & $0-21$ & $3.1(3.5)$ & $2.6(3.6)$ & 0.13 & 0.09 \\
\hline Diabetes-specific distress (PAID-20), mean (SD) & $0-100$ & $22.6(18.8)$ & $19.2(19.5)$ & 0.18 & $0.02 *$ \\
\hline
\end{tabular}

${ }^{*}$ Statistically significant, $p$-value $<0.05$

${ }^{a}$ Number of participants with available information

FFMQ-SF, Five Facet Mindfulness Questionnaire-Short Form; DHD15-index score, Dutch Healthy Diet 2015-index score; PHQ-9, Patient Health Questionnaire; GAD-7, Generalized Anxiety Disorder assessment; PAID-20, Problem Areas in Diabetes scale

by Cohen's $d$ for continuous variables and Cramer's $V$ for categorical variables. We used cutoffs of 0.20 for a small effect, 0.50 for medium effect, and 0.80 for large effect sizes (Cohen, 1988). To interpret the mediation analysis correctly, we first estimated the correlations of FFMQ-SF total and facet-specific scores with scores for emotional distress (including depressive symptoms, anxiety symptoms, and diabetes-specific distress) and correlations of emotional distress scores with the DHD15-index score, using Pearson's correlation coefficients $(r)$.

Univariable and multivariable linear regression models were conducted to estimate the association between the FFMQ-SF total score and the DHD15-index score, using a 1-point change as the unit. Model 1 was the unadjusted/ crude model. Model 2 was adjusted for demographic confounders, including age, gender, ethnicity, educational level, employment status, and partnership, which were selected a priori based on previous literature (Donofry et al., 2020; Tak et al., 2015). To examine the role of each mindfulness facet, both models were repeated by replacing the FFMQSF total score with each of the five facet-specific scores, namely observing, describing, acting with awareness, being non-judgmental, and being non-reactive.

Mediation analyses using depressive symptoms, anxiety symptoms, and diabetes-specific distress as potential mediators were performed for the FFMQ-SF total and facetspecific scores with the DHD15-index score. Standardized direct and indirect effects from the independent variable to the dependent variable via depressive symptoms, anxiety symptoms, and diabetes-specific distress were estimated by using the PROCESS macro for Social Science (Hayes, 2017). Path weights and confidence intervals for total effects were estimated using bootstrapping with 10,000 samples.

We repeated the analyses separately in participants with insulin-treated and non-insulin-treated T2DM to explore whether the estimated associations differed by diabetes management approach.

Tests were two-tailed and conducted at the 0.05 significance level. Data management was conducted in SAS software version 9.4 (SAS Institute, Cary, NC), and statistical analyses were performed using $\mathrm{R}$ version 3.6.1 and IBM SPSS Statistics 27.

\section{Results}

\section{Participants' Characteristics}

Means and SDs of FFMQ-SF total and facet-specific scores, the DHD15-index score, and emotional distress assessments were described separately by diabetes type (Table 2). No statistically significant difference in FFMQSF total and facet-specific scores, DHD15-index score, PHQ-9 scores, or GAD-7 scores was observed between T1DM and T2DM. According to the recommended cutoffs of PHQ-9 and GAD-7, 13.9\% of participants with T1DM had moderate depressive symptoms, and $5.4 \%$ had moderate anxiety, with similar percentages in participants with 
T2DM (13.2\% and 5.8\%, respectively). Nevertheless, participants with T1DM displayed a statistically significantly higher level of diabetes-specified distress as measured by the PAID-20 compared to participants with T2DM.

\section{Association between Mindfulness and Diet Quality}

In participants with T1DM (Table 3), total mindfulness, as measured with the FFMQ-SF total score, was associated with a higher diet quality $(\beta=0.15, p<0.01)$. This positive association was also observed for the facet-specific score observing $(\beta=0.16, p<0.01)$. After adjustment for demographic variables, the associations remained statistically significant ( $\beta=0.14, p=0.02$ for FFMQ-SF total score and $\beta=0.15, p=0.01$ for facet-specific score observing). Other facets of mindfulness were all slightly positively, but not statistically significantly, related to diet quality ( $\beta$ ranged from 0.06 to 0.08 ). In participants with T2DM (Table 3), FFMQ-SF total score $(\beta=0.12, p=0.03)$, the observing facet $(\beta=0.11, p=0.04)$, and being non-reactive $(\beta=0.11$, $p=0.04$ ) were positively related to the DHD15-index score in the crude model. However, these associations attenuated and became statistically non-significant after controlling for demographic variables (all $p>0.05$ ).

\section{Correlation between Dispositional Mindfulness, Emotional Distress, and DHD15-Index Score}

Across diabetes types, FFMQ-SF total score was statistically significantly correlated with lower depressive symptoms, anxiety symptoms, and diabetes-specific distress $(r:-0.56$ to -0.43 , all $p<0.05$; Supplemental Table 2). Most of the facet-specific scores, except for observing, were found to be statistically significantly negatively correlated with emotional distress. In participants with T1DM, observing facet was not statistically correlated with any emotional distress ( $r:-0.01$ to -0.09 , all $p>0.1$ ); however, in participants with T2DM, observing was significantly correlated with depressive symptoms $(r:-0.16, p<0.05)$ and diabetesspecific distress $(r:-0.19, p<0.05)$. As shown in Supplemental Table 3, emotional distress was correlated with a lower DHD15-index score $(r:-0.04$ to -0.05$)$, where a statistically significant correlation was observed only for depressive symptoms in both T1DM $(r:-0.14, p<0.05)$ and $\operatorname{T2DM}(r:-0.12, p<0.05)$.

\section{Mediation Effect of Emotional Distress}

Table 4 shows results from mediation analyses with depressive symptoms, anxiety symptoms, and diabetes-specific distress, respectively, for the FFMQ-SF total and facet-specific scores
Table 3 Linear regression models on the association between mindfulness and the Dutch Healthy Diet 2015-index score, stratified by diabetes type

\begin{tabular}{|c|c|c|c|c|c|c|}
\hline & \multicolumn{3}{|c|}{ Model 1} & \multicolumn{3}{|c|}{ Model 2} \\
\hline & $\beta$ & $t$ & $p$-value & $\beta$ & $t$ & $p$-value \\
\hline \multicolumn{7}{|l|}{ Type 1 diabetes } \\
\hline \multicolumn{7}{|l|}{ Mindfulness (FFMQ-SF) } \\
\hline Total & 0.15 & 2.66 & $<0.01 *$ & 0.14 & 2.30 & $0.02 *$ \\
\hline Observing & 0.16 & 2.77 & $<0.01^{*}$ & 0.15 & 2.53 & $0.01 *$ \\
\hline Describing & 0.09 & 1.63 & 0.10 & 0.06 & 0.94 & 0.35 \\
\hline Acting with awareness & 0.08 & 1.36 & 0.17 & 0.07 & 1.16 & 0.25 \\
\hline Being non-judgmental & 0.05 & 0.79 & 0.43 & 0.06 & 0.99 & 0.32 \\
\hline Being non-reactive & 0.11 & 1.83 & 0.07 & 0.08 & 1.38 & 0.17 \\
\hline \multicolumn{7}{|l|}{ Type 2 diabetes } \\
\hline \multicolumn{7}{|l|}{ Mindfulness (FFMQ-SF) } \\
\hline Total & 0.12 & 2.21 & $0.03 *$ & 0.08 & 1.52 & 0.13 \\
\hline Observing & 0.11 & 2.03 & $0.04 *$ & 0.05 & 1.04 & 0.30 \\
\hline Describing & 0.06 & 1.13 & 0.26 & 0.03 & 0.63 & 0.53 \\
\hline Acting with awareness & 0.09 & 1.68 & 0.09 & 0.07 & 1.29 & 0.20 \\
\hline Being non-judgmental & -0.01 & -0.17 & 0.86 & -0.01 & -0.14 & 0.89 \\
\hline Being non-reactive & 0.11 & 2.08 & $0.04 *$ & 0.09 & 1.76 & 0.08 \\
\hline
\end{tabular}

*Statistically significant, $p$-value $<0.05$

Model 1: Crude model

Model 2: Additionally adjust for age, gender, ethnicity, educational level, employment status, and partnership

$\beta$, standardized regression coefficients; $t$, coefficient divided by its standard error; $F F M Q-S F$, Five Facet Mindfulness Questionnaire-Short Form 
with the DHD15-index score. In both T1DM and T2DM, the standardized direct effect (c-path) of FFMQ-SF total score on DHD15-index score was not statistically significant in the presence of depressive symptoms as the mediator (T1DM: $\beta=0.11, p=0.11$; T2DM: $\beta=0.07, p=0.25$ ). However, the standardized indirect effect via depressive symptoms was not statistically significant. Similarly, no statistically significant standardized indirect effect via anxiety symptom or diabetes-specific distress was observed, while the direct effect of FFMQ-SF total scores on the DHD15-index remained statistically significant.

When further examining effects from specific facets of mindfulness in the presence of depressive symptoms, anxiety symptoms, or diabetes-specific distress, we found that, in T1DM, the direct effect from the facet-specific score observing remained statistically significantly related to DHD15index score, while the indirect effects of the emotional distress measures were not statistically significant. No statistically significant direct effect from other facet-specific scores was observed, whereas the indirect effects of facet-specific scores being non-judgmental and being non-reactive via depressive symptoms were statistically significant. In T2DM, no statistically significant indirect effect via either of the emotional distress scores was apparent for observing or being non-reactive in relation to DHD15-index, but there was a statistically significant indirect effect via depressive symptoms for describing and being non-judgmental.

\section{Sensitivity Analysis}

When examining participants with T2DM separately by treatment approach (i.e., insulin or no insulin), we observed that participants with T2DM using insulin were older and had a higher BMI and higher $\mathrm{HbA}_{1 \mathrm{c}}$, as well as longer diabetes duration, compared to those not using insulin. Nevertheless, there were no statistically significant differences in mindfulness scores, DHD15-index scores, or emotional distress scores between the two groups, and the effect sizes were relatively small (Supplemental Table 4). Among participants with insulin-treated T2DM, we observed no statistically significant associations for any of the mindfulness scores, whereas in those with non-insulin-treated T2DM, FFMQ-SF total score $(\beta=0.15, p=0.04)$ and being non-reactive $(\beta=0.17, p=0.02)$ were positively related to the DHD15-index score in the crude model, but these associations attenuated and became statistically non-significant after adjusting for demographic variables (all $p>0.05$ ) (Supplemental Table 5).

\section{Discussion}

In the present study, we found that a higher level of dispositional mindfulness and a higher score on observing were associated with higher diet quality. The results were more robust in people with T1DM. Our findings also suggest that overall mindfulness and the facet of observing are associated with higher diet quality in people with diabetes, independent of emotional distress.

The observed positive association between dispositional mindfulness and diet is in line with a recently published study in 406 healthy middle-aged adults (Donofry et al., 2020). This US study, to our knowledge, is the first to investigate dispositional mindfulness in relation to diet quality. They found that total mindfulness was positively associated with diet quality with effect estimates similar to our study ( $\beta=0.11, p=0.03)$. Diet quality was evaluated by a diet index, calculated based on American Dietary Guideline, comparable to the DHD15-index score used in our study. In the US study, mindfulness was examined as a unidimensional construct by the Mindful Attention Awareness Scale (MAAS) (Brown \& Ryan, 2003), while we used FFMQ-SF, which enabled a more detailed investigation into facets and thus added to the current scientific literature.

Given the multidimensional nature of dispositional mindfulness, we further examined the association of each of five facets of mindfulness by using FFMQ-SF facetspecific scores with diet quality to gain a better understanding of how specific skills cultivated in mindfulness can improve diet quality. Almost all facets tended towards a positive association with diet quality, which may have contributed to the statistically significant effect of total mindfulness. However, after adjustment for demographic characteristics, only the observing facet was found statistically significantly related to better diet quality in T1DM.

Although the associations between total mindfulness, the observing facet, and diet quality were positive in both T1DM and T2DM, statistical significance was observed in T1DM only. Such differences might be explained by the comparative importance of interoceptive observation for dietary choices in people with T1DM versus T2DM. For instance, T1DM usually has an early onset and important components in daily care involve monitoring blood level 24/7 and aligning and adjusting carbohydrate intake and insulin dosage accordingly. Therefore, those with a higher level of observing their interoceptive phenomena may be more likely to recognize the effects of consumed foods and drinks on their bodily sensations. Subsequently, they might be more cautious about keeping a healthier diet, such as a diet containing less sugar and fat, to manage their blood glucose levels. Another difference between T1DM 


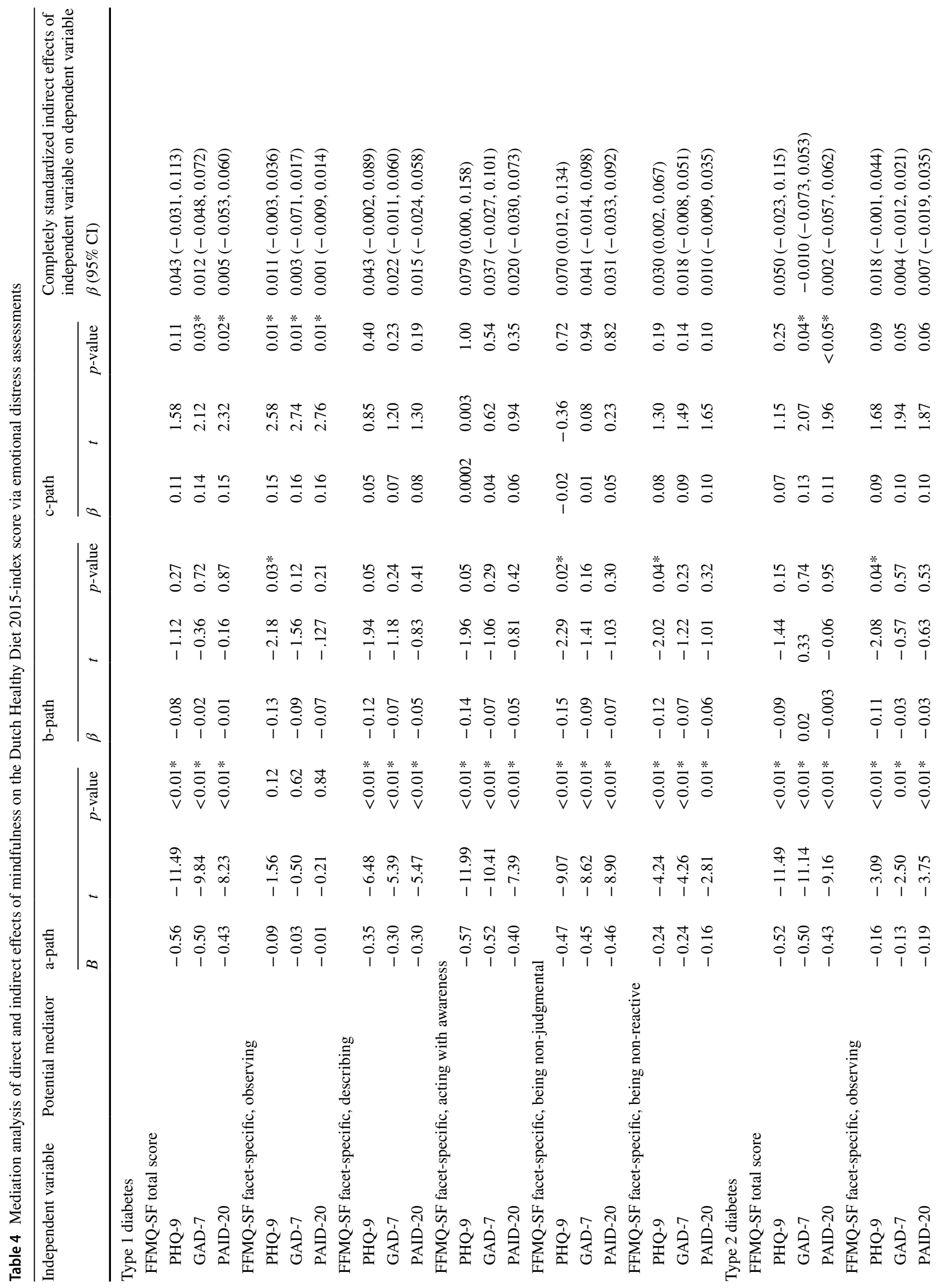




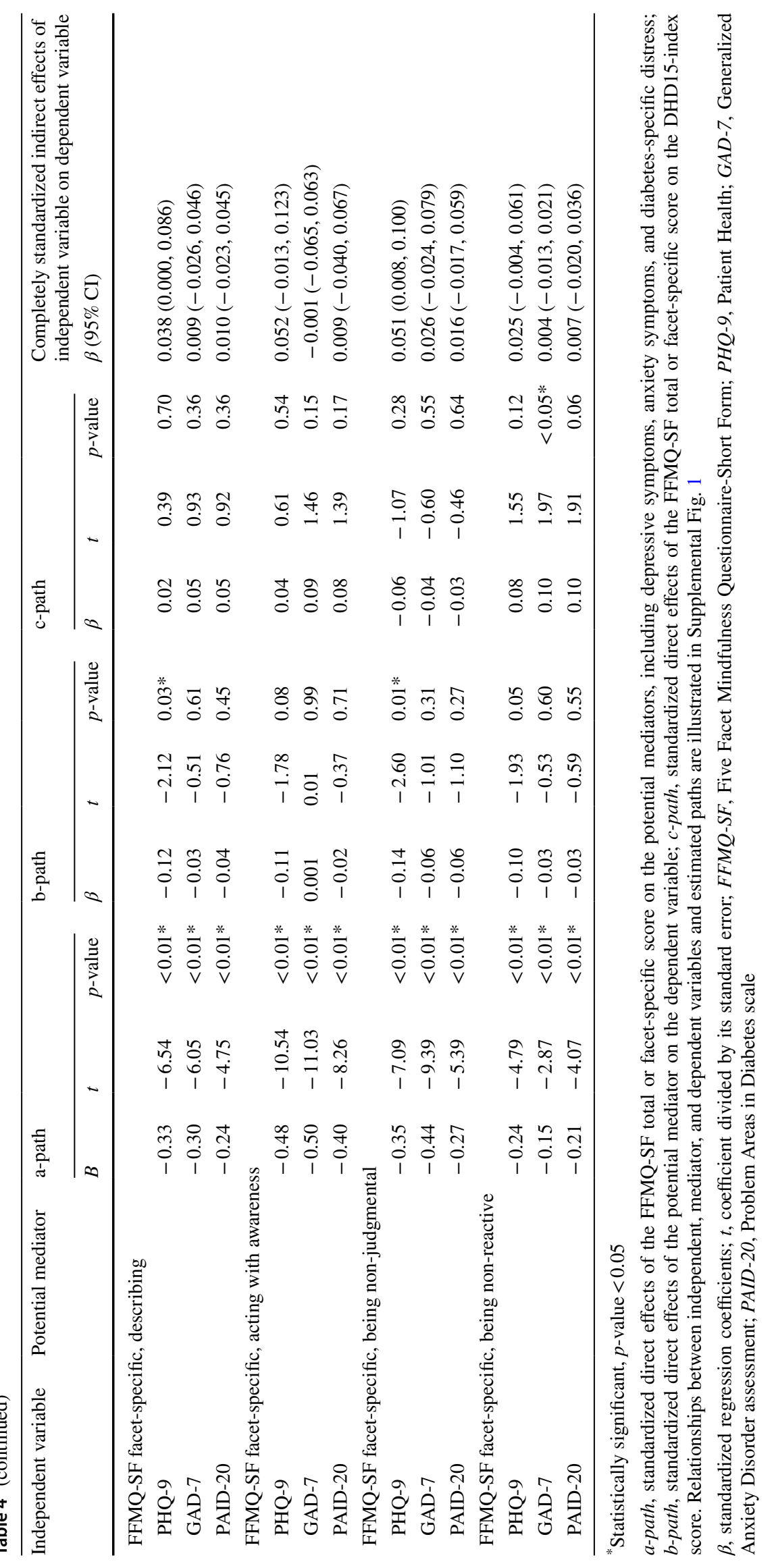


and $\mathrm{T} 2 \mathrm{DM}$ is that an unhealthy diet is a risk factor for the development of T2DM (IDF Diabetes Atlas, 2019). It is conceivable that for some people with T2DM, further improving diet quality is difficult to achieve and maintain.

Dissimilar to our hypothesis that all mindfulness facets would be related to higher diet quality, we observed no statistically significant associations for other mindfulness facets after adjusting for demographic characteristics. This finding seems to be inconsistent with the recent meta-analysis, where each mindfulness facet was found to be positively associated with healthy eating behaviors (Sala et al., 2020). However, it has to be kept in mind that diet quality, which is measured in our study, is a distinct concept and cannot be directly translated from healthy eating behaviors, which were assessed previously. Moreover, participants in our study had a relatively high diet quality, as reflected by a high mean DHD15-index score with small variation, which may also reduce the possibility of detecting potential associations for each facet.

The current findings regarding specific facets of mindfulness supported the notion that mindfulness is not a simple add-up of the facets in exerting effects on health and healthy behaviors (Baer et al., 2006). Future studies are warranted to gain a better understanding of the facet-specific effects of mindfulness in the different health-related contexts, including healthy behaviors in different populations, such as consuming healthy diets.

Mediation analyses showed that total mindfulness is related to diet quality, independently of emotional distress. The inclusion of emotional distress measures did not change the association between mindfulness and diet quality, nor did it mediate that association. This finding is somewhat different from the US study (Donofry et al., 2020), where such a statistical mediation by depressive symptoms was observed. The US study reported that their participants, in general, had mild depressive symptoms, using the Centre for Epidemiologic Studies Depression Scale (mean value of 8.93 and SD of 8.25 ) and $17.7 \%$ had clinically significant depressive symptoms. However, in our study, moderate depressive symptoms were present in $13.9 \%$ and $13.2 \%$ of participants with T1DM and T2DM, respectively. Moreover, the relationship between mindfulness and depression is complicated and might be bidirectional. It is plausible that people who develop depressive symptoms may subsequently be less mindful and vice versa. However, when examining the facet-specific score, we found a statistically significant indirect effect via depressive symptoms for being non-judgmental and being non-reactive in those with T1DM and for describing and being non-judgmental in those with T2DM. These observations indicate that the potential mediation role of depressive symptoms is unneglectable when investigating the effect of mindfulness on diet quality. Thus, longitudinal studies are needed for a better understanding of these associations.

\section{Limitations and Future Research}

Several limitations need to be addressed. Due to the crosssectional and observational nature of the study, we could not draw any conclusions regarding causality. Another important limitation is that dietary data were obtained from a nonvalidated FFQ, which led to a lack of information on portion size and certain food items, such as tea, coffee, and legumes. Moreover, our study sample may not be a good representation of the general Dutch diabetes population. Given the media-based recruitment and online survey method, our participants may be more active in their diabetes management. This explains the generally high diet quality, indicated by a high DHD15-score, with a fairly low variation among participants, as well as the relatively low scores of emotional distress. This may have contributed to the relatively small effect sizes and limited power to identify possible mediation effects by emotional distress.

Findings from this cross-sectional study suggest that mindfulness, especially observing facet, may relate to a healthier diet in adults with diabetes. Future longitudinal and intervention studies, incorporating more thorough assessments on dietary intakes, would contribute to a more comprehensive understanding of the association.

Supplementary Information The online version contains supplementary material available at https://doi.org/10.1007/s12671-021-01754-x.

Acknowledgements The authors thank all the study participants for volunteering their time and experiences. They also thank the Dutch Diabetes Association and the Dutch Diabetes Research Foundation for their kind and very valuable assistance in the recruitment of participants.

Author Contribution The Diabetes MILES Study is an international collaborative established in 2011, jointly led by FP and JS. GN, FP, JS, and MB collected the data. SL and SSM conceived and designed the research question and developed the analysis plan. SL and SSM had full access to all the data and conducted the data analyses. SL and SSM wrote the first draft of the paper. All authors interpreted the data and contributed to the writing of the paper. All authors revised several versions of the manuscript and approved the final version for submission. SL and SSM act as guarantors. The corresponding author attests that all listed authors meet authorship criteria and that no others meeting the criteria have been omitted.

Funding Open access funding provided by Karolinska Institute. The data collection for the present study was supported by the Prof. Dr. J. Terpstra Young Investigator Award 2010 from the Dutch Association for Diabetes Research (Nederlandse Vereniging voor Diabetes Onderzoek)/Lilly Diabetes, awarded to GN. JS is supported by core funding to the Australian Centre for Behavioural Research in Diabetes provided by the collaboration between Diabetes Victoria and Deakin University. The funding source had no role in the design, data collection, analysis, 
or interpretation of the study or in the decision to submit the manuscript for publication.

Data Availability The data that were analyzed for the present study will be made available upon request.

\section{Declarations}

Ethics Approval and Consent Diabetes MILES-The Netherlands was approved by the Psychological Research Ethics Committee of Tilburg University (EC-2011 5) and digital informed consent was obtained from all participants included in the study.

Conflict of Interest The authors declare no competing interests.

Open Access This article is licensed under a Creative Commons Attribution 4.0 International License, which permits use, sharing, adaptation, distribution and reproduction in any medium or format, as long as you give appropriate credit to the original author(s) and the source, provide a link to the Creative Commons licence, and indicate if changes were made. The images or other third party material in this article are included in the article's Creative Commons licence, unless indicated otherwise in a credit line to the material. If material is not included in the article's Creative Commons licence and your intended use is not permitted by statutory regulation or exceeds the permitted use, you will need to obtain permission directly from the copyright holder. To view a copy of this licence, visit http://creativecommons.org/licenses/by/4.0/.

\section{References}

Ahola, A. J., Mikkilä, V., Mäkimattila, S., Forsblom, C., Freese, R., Groop, P. H., \& FinnDiane Study Group. (2012). Energy and nutrient intakes and adherence to dietary guidelines among Finnish adults with type 1 diabetes. Annals of Medicine, 44(1), 73-81

Baer, R. A., Smith, G. T., Hopkins, J., Krietemeyer, J., \& Toney, L. (2006). Using self-report assessment methods to explore facets of mindfulness. Assessment, 13(1), 27-45.

Barney, J. L., Murray, H. B., Manasse, S. M., Dochat, C., \& Juarascio, A. S. (2019). Mechanisms and moderators in mindfulness-and acceptance-based treatments for binge eating spectrum disorders: A systematic review. European Eating Disorders Review, 27(4), 352-380.

Barrington, W. E., Beresford, S. A., McGregor, B. A., \& White, E. (2014). Perceived stress and eating behaviors by sex, obesity status, and stress vulnerability: Findings from the vitamins and lifestyle (VITAL) study. Journal of the Academy of Nutrition and Dietetics, 114(11), 1791-1799.

Beshara, M., Hutchinson, A. D., \& Wilson, C. (2013). Does mindfulness matter? Everyday mindfulness, mindful eating and selfreported serving size of energy dense foods among a sample of South Australian adults. Appetite, 67, 25-29.

Bohlmeijer, E., Ten Klooster, P. M., Fledderus, M., Veehof, M., \& Baer, R. (2011). Psychometric properties of the five facet mindfulness questionnaire in depressed adults and development of a short form. Assessment, 18(3), 308-320.

Brown, K. W., \& Ryan, R. M. (2003). The benefits of being present: Mindfulness and its role in psychological well-being. Journal of Personality and Social Psychology, 84(4), 822.

Cohen, J. (1988). Statistical power analysis for the behavioral sciences. Academic press.
Donofry, S. D., Erickson, K. I., Levine, M. D., Gianaros, P. J., Muldoon, M. F., \& Manuck, S. B. (2020). Relationship between dispositional mindfulness, psychological health, and diet quality among healthy midlife adults. Nutrients, 12(11), 3414.

Estruch, R., Ros, E., Salas-Salvadó, J., Covas, M. I., Corella, D., Arós, F., ... \& Martínez-González, M. A. (2013). Primary prevention of cardiovascular disease with a Mediterranean diet. New England Journal of Medicine, 368(14), 1279-1290

Forouhi, N. G., Misra, A., Mohan, V., Taylor, R., \& Yancy, W. (2018). Dietary and nutritional approaches for prevention and management of type 2 diabetes. Bmj, 361 .

Glasgow, R. E., McCaul, K. D., \& Schafer, L. C. (1986). Barriers to regimen adherence among persons with insulin-dependent diabetes. Journal of Behavioral Medicine, 9(1), 65-77.

Hayes, A. F. (2017). Introduction to mediation, moderation, and conditional process analysis: A regression-based approach. Guilford publications.

International Diabetes Federation. IDF Diabetes Atlas, 9th edn. Brussels, Belgium: International Diabetes Federation, 2019.

Jordan, C. H., Wang, W., Donatoni, L., \& Meier, B. P. (2014). Mindful eating: Trait and state mindfulness predict healthier eating behavior. Personality and Individual Differences, 68, 107-111.

Kabat-Zinn, J. (2009). Full catastrophe living: Using the wisdom of your body and mind to face stress, pain, and illness. Delta.

Kroenke, K., Spitzer, R. L., \& Williams, J. B. (2001). The PHQ9: Validity of a brief depression severity measure. Journal of General Internal Medicine, 16(9), 606-613.

Ley, S. H., Hamdy, O., Mohan, V., \& Hu, F. B. (2014). Prevention and management of type 2 diabetes: Dietary components and nutritional strategies. The Lancet, 383(9933), 1999-2007.

Liday, C., \& Kirkpatrick, C. (2019). Optimal dietary strategies for prevention of atherosclerotic cardiovascular disease in diabetes: Evidence and recommendations. Current Cardiology Reports, 21(11), 1-10.

Looman, M., Feskens, E. J., de Rijk, M., Meijboom, S., Biesbroek, S., Temme, E. H., de Vries, J., \& Geelen, A. (2017). Development and evaluation of the Dutch Healthy Diet index 2015. Public Health Nutrition, 20(13), 2289-2299.

Mason, A. E., Epel, E. S., Kristeller, J., Moran, P. J., Dallman, M., Lustig, R. H., Acree, M., Bacchetti, P., Laraia, B. A., Hecht, F. M., \& Daubenmier, J. (2016). Effects of a mindfulness-based intervention on mindful eating, sweets consumption, and fasting glucose levels in obese adults: Data from the SHINE randomized controlled trial. Journal of Behavioral Medicine, 39(2), 201-213.

Mikolajczyk, R. T., El Ansari, W., \& Maxwell, A. E. (2009). Food consumption frequency and perceived stress and depressive symptoms among students in three European countries. Nutrition Journal, 8(1), 1-8.

Miller, C. K. (2017). Mindful eating with diabetes. Diabetes Spectrum, 30(2), 89-94.

Miller, C. K., Kristeller, J. L., Headings, A., \& Nagaraja, H. (2014). Comparison of a mindful eating intervention to a diabetes selfmanagement intervention among adults with type 2 diabetes: A randomized controlled trial. Health Education \& Behavior, 41(2), 145-154.

Nefs, G., Bot, M., Browne, J. L., Speight, J., \& Pouwer, F. (2012). Diabetes MILES-The Netherlands: Rationale, design and sample characteristics of a national survey examining the psychosocial aspects of living with diabetes in Dutch adults. BMC Public Health, 12(1), 1-11.

Patterson, R. E., Haines, P. S., \& Popkin, B. M. (1994). Diet quality index: Capturing a multidimensional behavior. Journal of the American Dietetic Association, 94(1), 57-64. 
Richardson, A., Adner, N., \& Nordström, G. (2001). Persons with insulin-dependent diabetes mellitus: Acceptance and coping ability. Journal of Advanced Nursing, 33(6), 758-763.

Sala, M., Rochefort, C., Lui, P. P., \& Baldwin, A. S. (2020). Trait mindfulness and health behaviours: A meta-analysis. Health Psychology Review, 14(3), 345-393.

Senécal, C., Nouwen, A., \& White, D. (2000). Motivation and dietary self-care in adults with diabetes: Are self-efficacy and autonomous self-regulation complementary or competing constructs? Health Psychology, 19(5), 452.

Shultz, J. A., Sprague, M. A., Branen, L. J., \& Lambeth, S. (2001). A comparison of views of individuals with type 2 diabetes mellitus and diabetes educators about barriers to diet and exercise. Journal of Health Communication, 6(2), 99-115.

Snoek, F. J., Pouwer, F., Welch, G. W., \& Polonsky, W. H. (2000). Diabetes-related emotional distress in Dutch and US diabetic patients: Cross-cultural validity of the problem areas in diabetes scale. Diabetes Care, 23(9), 1305-1309.

Spitzer, R. L., Kroenke, K., Williams, J. B., \& Löwe, B. (2006). A brief measure for assessing generalized anxiety disorder: The GAD-7. Archives of Internal Medicine, 166(10), 1092-1097.

Tak, S. R., Hendrieckx, C., Nefs, G., Nyklíček, I., Speight, J., \& Pouwer, F. (2015). The association between types of eating behaviour and dispositional mindfulness in adults with diabetes. Results from Diabetes MILES. The Netherlands. Appetite, 87, 288-295.

van Son, J., Nyklíček, I., Nefs, G., Speight, J., Pop, V. J., \& Pouwer, F. (2015). The association between mindfulness and emotional distress in adults with diabetes: Could mindfulness serve as a buffer? Results from Diabetes MILES: The Netherlands. Journal of Behavioral Medicine, 38(2), 251-260.

Vogtschmidt, Y. D., Nefs, G., Speight, J., Bot, M., Pouwer, F., \& Soedamah-Muthu, S. S. (2020). Depressive and anxiety symptoms and following of the Dutch Dietary Guidelines 2015 in adults with diabetes: Results from Diabetes MILES-The Netherlands. Journal of Psychosomatic Research, 135, 110160.

Westenbrink, S., Jansen-van der Vliet, M., \& van Rossum, C. (2012). Updated folate data in the Dutch Food Composition Database and implications for intake estimates. Food \& Nutrition Research, $56(1), 5449$.

Publisher's Note Springer Nature remains neutral with regard to jurisdictional claims in published maps and institutional affiliations. 